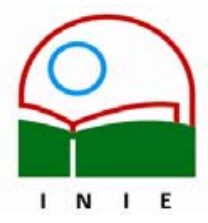

Universidad de Costa Rica

Facultad de Educación

Instituto de Investigación en Educación

ACTUALIDADES INVESTIGATIVAS EN EDUCACION

\title{
ACERCAMIENTO PARADIGMÁTICO DE LA AUTOEVALUACIÓN COMO INVESTIGACIÓN
}

\author{
Silvia Chacón Ramírez ${ }^{1}$
}

\begin{abstract}
Resumen: Este artículo es el primero de una secuencia, cuya intención es abordar el tema de la autoevaluación. Este tópico es llevado al plano de la investigación, con el propósito de lograr una comprensión de lo que se hace, en el entendido de que las intenciones de la autoevaluación pueden variar entre el control externo y la búsqueda del bien común, éste último estatutariamente establecido en la Universidad de Costa Rica. El contexto para este artículo se ubica en el proceso de autoevaluación desarrollado en la Escuela de Formación Docente de la Facultad de Educación.

Adicionalmente, se presentan en este artículo algunas premisas y cuestionamientos que develan en alguna medida, lo ideológico y epistemológico de un proceso de autoevaluación, además se incluye un análisis que permite afirmar que la tendencia paradigmática en la universidad, respecto a la autoevaluación, corresponde a una fluctuación entre lo naturalista y lo positivista.

A manera de conclusión se establece la necesidad de develar las razones que subyacen en los procesos de autoevaluación, que conlleven a dos situaciones, la primera, comprender el por qué se hace lo que hace a fin de visualizar los alcances de las acciones y segunda, lograr un compromiso con aquellas intenciones más coherentes que permitan a la educación como práctica social, lograr sus metas de transformación. Finalmente se presentan otros cuestionamientos que permanecen sin resolver.
\end{abstract}

\section{Palabras clave: AUTOEVALUACIÓN/ EDUCACIÓN SUPERIOR/ INVESTIGACIÓN EVALUATIVA/ PARADIGMAS INVESTIGATIVOS/}

\begin{abstract}
This is the first article of a sequence, which intends to tackle auto evaluation issues. This topic is brought to the research field to comprehend what is being done, considering that it purposes may vary between external control and common welfare lawfully established at the Universidad de Costa Rica. This article is within the context of the auto evaluation process developed at the Teachers and Lecturers Formation School, Education Faculty.

Additionally, some premises and questions are presented. These premises and questions show somehow ideology and epistemology underneath auto evaluation. An analysis is included which permits to state that the paradigmatic trend at the university with respect to auto evaluation fluctuates between naturalism and positivism. As a conclusion three issues are discussed. First, the need to discover subjacent reasons in auto evaluation processes is established. Second, comprehend why what is being done, is done, to visualize actions outreach. And third, the commitments with those coherent intentions which allow education, as a social practice, to achieve its transformation goals. Finally, unsolved questions and issues are also presented.
\end{abstract}

Key words: SELF-EVALUATION/ COLLEGE GRADE EDUCATION/ EVALUATION RESEARCH/ RESEARCH PARADIGMS/

\footnotetext{
1 Maestría Profesional en Planificación Curricular y Licenciatura en Educación Preescolar ambos títulos de la Universidad de Costa Rica. Profesora de la Escuela de Formación Docente de la misma universidad en los campos de Educación Preescolar, principalmente en las áreas de Artes del Lenguaje, Experiencia Profesional Docente, Literatura Infantil, Desarrollo de la Creatividad, Investigación. La labor investigativa más reciente se ubica en los campos autoevaluación y la Educación Ambiental.
}

Correo electrónico: schacon@cariari.ucr.ac.cr

Artículo recibido: 18 de abril, 2005

Aprobado: 20 de junio, 2005 


\section{Introducción}

La llegada del siglo XXI encontró a la Universidad de Costa Rica en una labor de promoción de cultura evaluativa en sus carreras, como respuesta, principalmente, a dos demandas, la primera de carácter político-económico: la rendición de cuentas; la segunda, más social: brindar evidencia del nivel de calidad respecto a una educación superior que se debe, por principio estatutario, al bien común y a la transformación social.

Este interés en el tema nace de la "inmersión" profesional y personal, en un proceso de reflexión y sistematización, que tiene como punto de partida el desarrollo de una compleja autoevaluación iniciada en el año 1999 en la Escuela de Formación Docente, instancia dedicada a la formación de docentes de educación preescolar, primaria, secundaria y capacitación para la docencia universitaria.

El presente artículo corresponde a una de varias entregas que se realizarán, para abordar el tema de la autoevaluación como investigación en esta Escuela.

La responsabilidad de dicha investigación recayó en una comisión integrada por tres personas, la profesora Ana Cecilia Hernández, la profesora Carmen Liddy Fallas y quien suscribe, a quien le correspondió asumir un doble papel, por una parte ser la coordinadora de comisión de autoevaluación y por el otro, cumplir como investigadora principal.

Bajo el convencimiento de que los procesos de autoevaluación deben enmarcarse al amparo de la investigación, es que en esta primera entrega se aborda la posición paradigmática. En una segunda entrega se ofrecerá una caracterización metodológica, desde los enfoques cuantitativo y cualitativo del proceso desarrollado específicamente en la Escuela de Formación Docente, durante los años 1999 al 2003. Una tercera entrega estaría enfocada al proceso metodológico como tal, con sus aciertos y desaciertos, dando respuesta a una gran pregunta, ¿cómo se aborda una autoevaluación simultánea de tres carreras, dos de ellas con miras a la acreditación? Esta situación es única hasta el momento en la Universidad de Costa Rica.

En este contexto de intenciones, el presente artículo incursiona en los siguientes aspectos:

- La investigación evaluativa: un marco de referencia general

- La evaluación educativa y la autoevaluación

- La autoevaluación transformada en investigación.

- La autoevaluación como segundo objeto de estudio.

- Una posición paradigmática de investigación para la autoevaluación.

- Consideraciones finales 


\section{La investigación evaluativa: un marco de referencia general}

Se considera que la investigación evaluativa es parte de la investigación educativa. Más específicamente, tiene su interés en la práctica educativa para obtener su cambio, es decir es una investigación que se orienta hacia lo político.

Arnal, de Rincón y de la Torre (en Díaz, 1996), consideran que el diseño de este tipo de investigación debe contribuir a solucionar problemas o aportar directrices para la acción, en este sentido señalan la necesidad de describir con amplitud la complejidad de las situaciones, con el propósito de establecer marcos conceptuales que permitan la comprensión y la toma de decisiones.

Para los mismos autores el propósito principal es determinar la eficacia de las organizaciones y programas educativos, así como contribuir al desarrollo de una perspectiva teórica.

Por su parte, para Cook y Reichardt, la investigación evaluativa se acerca tanto al paradigma cuantitativo como cualitativo, sin embargo, afirman que por largo tiempo lo cuantitativo ha sido predominante, ante lo cual valorizan desde la perspectiva cualitativa, al señalar que es preciso "(...) mantener la evaluación próxima a los mundos contextuales de los afectados por ésta" (1997, p. 73). Agregan que los procedimientos cuantitativos permiten la posibilidad de generalizar los hallazgos, pero que es importante recoger múltiples perspectivas y emplear métodos múltiples para captar la visión más global de la intervención social.

Estos mismos autores consideran que durante el proceso de investigación evaluativa se hace necesario registrar la historia del proceso de evaluación (acontecimientos, cambios, etc.), en que se incursiona y enfatizar en que ésta es una tarea relacionada con la propia evaluación.

Díaz (1996) ha propuesto algunas características concernientes a este tipo de investigación, las cuales se resumen a continuación:

- Sus resultados no se pueden aplicar a otros programas, pues ésta se realiza en una situación específica y en un momento determinado.

- Se dificulta y a veces resulta inadecuado, formular hipótesis precisas.

- Los datos están condicionados por la viabilidad del proceso, es decir se presenta la disyuntiva entre contar con datos accesibles y aquello que desean los evaluadores y administradores del programa... 
- El control de muchas de las variables de importancia suele ser superficial. Resulta difícil conseguir la técnica de la aleatorización, cuyo fin primordial es contrarrestrar la influencia de las variables sobre la varianza sistemática.

- El criterio para decidir si continuar, modificar, ampliar o sustituir el programa, recae sobre la institución responsable del programa o de la audiencia a que va dirigido el informe de evaluación.

- El informe de la evaluación debe sujetarse a las exigencias de quien toma las decisiones sobre el programa.

Al igual que Cook y Reichardt, Díaz (1996) considera que debe utilizarse una extensa variedad de métodos cuyo fin primordial es proveer información sobre asuntos que se han planteado en los programas educativos, con el propósito de facilitar la toma de decisiones. Complementa esta idea, señalando que los paradigmas no se consideran como determinantes exclusivos de las metodologías que requieren utilizarse y que por el contrario, puede emplearse una serie de métodos asociados a cada paradigma. De tal manera, los diseños de investigación evaluativa servirán como marcos generales de referencia para planificar una investigación de esta índole.

Ahora bien, Cook y Reichardt (1997, p. 136), enfatizan en que "(...) la tarea de examinar, definir y redefinir el entorno institucional en educación debe ser responsabilidad principal del propio gremio". Es aquí donde surge la "autoevaluación" como modalidad de investigación evaluativa.

Particularmente para el Sistema Nacional de Acreditación (2000, p. 15) la autoevaluación:

Es un proceso previo a la acreditación que debe realizar la carrera o programa que tiene intención de acreditarse. Debe tener características que den fe de la formalidad y rigor técnico con se haya realizado y la información obtenida debe ser válida, confiable y verificable.

Debe ser un proceso participativo de revisión sistemática del quehacer de la carrera o programa, organizado por un equipo de trabajo comprometido y calificado. (Sistema Nacional de Acreditación, 2000, p. 15)

Ahora bien, para el comité responsable de la autoevaluación en la Escuela de Formación Docente, ésta corresponde al compromiso asumido por la Asamblea de Escuela 
para revisar sus planes de formación, en relación con aquellos factores y criterios ya definidos por el Centro de Evaluación Académica, instancia encargada de asesorar los procesos de autoevaluación en la Universidad de Costa Rica. Tal compromiso implica participar del proceso, asumir los resultados y proponer soluciones que permitan el mejoramiento del quehacer y por tanto, el cumplimiento de la misión, propuesta en los fundamentos del proyecto pedagógico de la Escuela de Formación Docente.

Tabla 1 Factores y criterios emitidos por el Centro de Evaluación Académica.

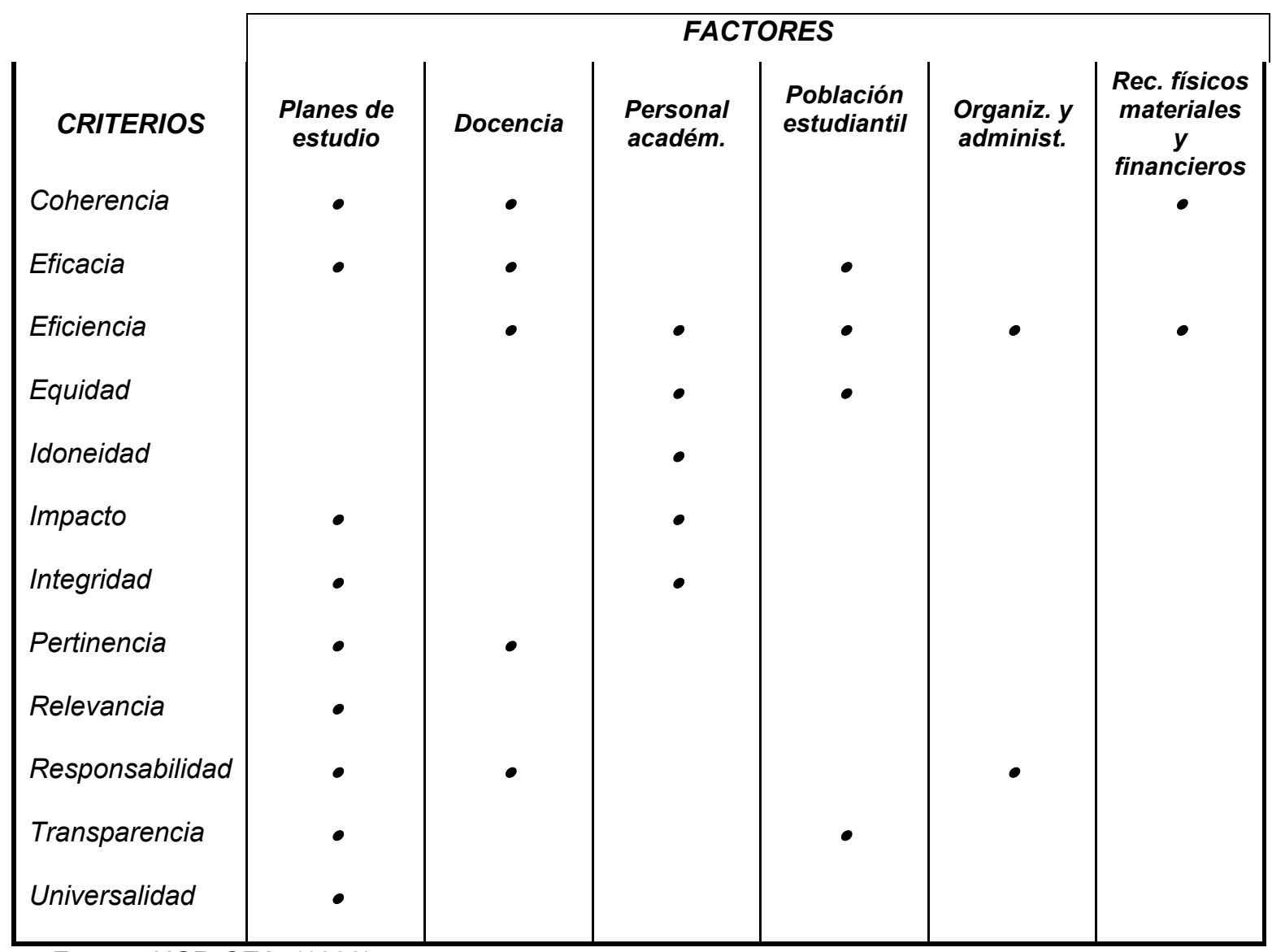

Fuente: UCR-CEA. (1999)

De esta forma, el Centro de Evaluación Académica de la Universidad de Costa Rica, regularmente utiliza a su vez, los criterios definidos por el Sistema Nacional de Acreditación: coherencia, eficacia, eficiencia, equidad, idoneidad, impacto, integridad, pertinencia, relevancia, responsabilidad, transparencia y universalidad, los cuales se encuentran definidos en su Guía de Autoevaluación, versión del año 1999. 


\section{La evaluación educativa y la autoevaluación}

Para establecer la relación entre evaluación educativa y autoevaluación, se observan en este apartado tres puntos de vista, uno contextual o axiológico, el otro de control externo o economicista y, el tercero, un punto de vista evolutivo.

\subsection{Punto de vista contextual o axiológico}

Una de las mejores representaciones de este punto de vista se encuentra en, de Alba (1991), quien concibe la evaluación educativa como una acción hermenéutica y epistemológica, además caracteriza la evaluación como un proceso complejo, que conlleva no sólo a la reflexión y el análisis crítico, sino a una síntesis conceptual valorativa. Hace ver que esta síntesis es fundamental para poder conocer y evaluar el origen y desarrollo de procesos o situaciones sociales, así como la confrontación estructural que se da entre ambos.

Adicionalmente, para esta autora la evaluación tiene un carácter axiológico, lo cual implica comprender pero además, "implica el confundir esferas tan importantes del conocimiento y el saber, (en un sentido kantiano); esto es confundir la facultad del entendimiento con la del pensamiento, la razón pura con la razón práctica" (de Alba, 1991, p.98). Señala además la problemática que conlleva el establecimiento del cuerpo valorativo en los procesos de evaluación; sin embargo ofrece una orientación, al señalar tres sentidos a los cuales dirigirse, los cuales, en términos concretos corresponden a:

- Revisión de los valores básicos o fundamentales (considerados como inamovibles)

- Valoración del sustento o los sustentos teóricos de la evaluación

- Compromiso mismo de la instancia evaluadora.

Particularmente, desde la evaluación curricular, de Alba (1991) anota que al ser el currículum un proceso social, hay que pensarlo desde la perspectiva de la totalidad social y que por tanto los análisis particulares, así como la delimitación de aspectos deben partir de la totalidad social. Enfáticamente la autora afirma que:

Detrás del carácter científico y objetivo de la evaluación subyace un problema de elaboración de juicio de valor sobre el objeto a evaluar, problema complejo tanto en su esencia como en sus dimensiones; la evaluación tiene un carácter axiológico ineludible que se complejiza en sus dimensiones social, económica, política, ideológica (de Alba, 199, p. 88) 
Agrega la misma autora, que es el carácter axiológico lo que la convierte a la evaluación en una actividad humana con el máximo nivel de complejidad y responsabilidad, ante el ejercicio conciente de razonamiento.

Ahora bien, desde la perspectiva que presenta de Alba, se puede abordar entonces la temática de la autoevaluación, al considerar que por lo regular, los procesos autoevaluativos nacen del convencimiento interno, grupal y genuino de un grupo de personas, cuyo deseo es determinar el alcance de la propia práctica educativa, llámese curso, carrera o plan de estudio. Éste convencimiento se orienta hacia la toma de conciencia de que lo que se hace debe mejorarse para responder a las necesidades y demandas sociales.

La sensibilización y la conciencia grupal van a determinar un compromiso participativo, no para la búsqueda de reconocimiento como tal, sino para trascender a una genuina transformación. Aquí el convencimiento grupal determina una consecuencia grupal pero también individual, sea desde un nivel u otro, para ese tipo de autoevaluación se perfila un alto nivel de mejoramiento de la práctica educativa, por cuanto se tiende a clarificar el objeto de acción, su propósito y la forma cómo se aborda la práctica educativa, es decir se llega a una conciencia, social-política y epistemológica de dicha práctica.

La autoevaluación, por tanto, no tendrá carácter aséptico, sino que corresponde a un proceso complejo, tamizado por un cuerpo axiológico, que la convierte en una actividad humana, con errores y aciertos; divergencias y convergencias.

\subsection{Punto de vista de control externo o economicista.}

Desde este punto de vista, según Díaz Barriga (1996), se reconocen para la evaluación educativa, influencias de corte internacional, que presionan por una exigencia técnica de proporcionar información, tanto al sistema, como a aquellas personas que toman decisiones y finalmente a la sociedad. La evaluación educativa se va imponiendo como una tendencia globalizadora. Dentro de esta línea se encuentra se enmarca el criterio de la CEPAL-UNESCO, al señalar que:

(...) la evaluación es eminentemente una práctica estatal, es una manifestación del tránsito entre el Estado Benefactor y el Estado Evaluador. Esta nueva función se lleva a cabo con la finalidad de analizar el cumplimiento de las metas del sector educativo, difundir información sobre el funcionamiento del sistema educativo. (Díaz Barriga, 1996, p. 15) 
Bajo esta visión la autoevaluación responde a fuerzas externas, una solicitud o exigencia externa para una instancia educativa. El propósito es rendir cuentas; hay un alto sentido en los criterios de eficiencia y eficacia como determinantes de presupuestos económicos. Podría decirse que el involucramiento de las personas responsables de la autoevalución es tan importante como su labor coordinadora del proceso. El convencimiento de mejorar la práctica educativa no se gesta a lo interno, sino que por el contrario, responde a una solicitud o mandato, que puede darse en un momento oportuno o no.

Desde esta perspectiva de control externo, existe un ente evaluador que determina los criterios por evaluar y que en poca medida pueden ser variados por el grupo responsable de la evaluación. Según Guba y Lincoln (1989), este tipo de evaluación corresponde a una evaluación de "tercera generación", como se verá más adelante.

El dilema o la dicotomía se debe al encuentro de estos dos puntos de vista, el primero, representado por De Alba, que responde a un acontecer cotidiano, de largo transcurrir y que corre el riesgo de no traducirse en cambios claros para el mejoramiento de la práctica, el segundo, de control externo, que limita la reflexión sobre las implicaciones del proceso de autoevaluación y sus resultados, ya sea a nivel grupal o a nivel personal. Bajo esta perspectiva se dificulta determinar como se afecta cada persona involucrada.

Dos criterios son básicos ante este dilema, por una parte la absoluta conciencia del para qué se realiza un proceso evaluativo y segundo, observar el proceso desde una perspectiva crítica o autocrítica. Este segundo criterio llevaría implícita una conciencia epistémica de lo que sucede en el proceso evaluativo. La resolución de este dilema es una interesante y relevante situación sobre todo en nuestro contexto de país latinoamericano, influenciado y sometido a criterios económicos y globalizantes.

\subsection{Punto de vista evolutivo}

Continuando con los puntos de vista sobre la autoevalución, se presenta una tercera perspectiva de análisis, esto es, el carácter evolutivo de la evaluación. Esta perspectiva la presentan Guba y Lincoln (1989), quienes distinguen cuatro generaciones de cambio en los procesos evaluativos. La primera centrada en el "rendimiento escolar", la segunda "descriptiva", la tercera "de juicio" y la cuarta "constructiva". A continuación se explican y posteriormente se establece una relación respecto a la autoevaluación.

- La primera generación, ubica la calidad de la educación en el rendimiento educativo. El papel del evaluador representa un técnico altamente experimentado en la elaboración de instrumentos, para ejercer la práctica de una evaluación externa. 
- La segunda generación, de "evaluación descriptiva", tiene origen posteriormente a la Primera Guerra Mundial, Ilega a considerar insuficiente el "rendimiento del estudiante" como único criterio de calidad. Surge entonces la descripción de patrones que evidencien las fortalezas y debilidades, en relación con los objetivos establecidos. Sin dejar de lado el papel técnico, el evaluador pasa a describir el logro de objetivos. También esta situación corresponde a una evaluación externa.

- La tercera generación se denomina "de juicio", porque incluye el juicio de valor como parte fundamental del acto de evaluar. Esta posición surge posteriormente al lanzamiento del Sputnik en Rusia, en el año 1957; agrega al evaluador un papel de juez, además de las funciones señaladas en las otras dos generaciones. El juicio de valor corresponde a una interpretación particular ofrecida sobre la base de estándares de comparación, los cuales se valoran dentro de una connotación científica.

Desde el punto de vista de evaluación curricular, esta tercera generación evidencia claramente un "enfoque tecnológico". Críticamente, de Alba, afirma que bajo este enfoque se...

(...) pretende validar y legitimar las decisiones que se toman en relación a la evaluación, o sea que detrás del pretendido carácter científico de la evaluación se esconde una necesidad de quienes postulan el discurso, de legitimar las prácticas educativas, necesidad que va cobrando una fuerza cada vez mayor (de Alba, 1991, p. 81).

Se implica por tanto, que dentro de esta generación se ubica la autoevaluación para la acreditación. Según Dobles (1996), la acreditación surge en el contexto estadounidense norteamericano, a principios del siglo XX. Es así como a partir de la acreditación de escuelas secundarias se desprende la acreditación universitaria. El año 1906 señala la época de expansión de la acreditación, cuya intención fue la ubicación de estudiantes que se trasladaban de una universidad a otra, es cuando surgen los primeros estándares.

En un sentido tradicional, a la acreditación se la considera desde una relación vertical entre un organismo externo (la comisión acreditadora), como ente activo que preestablece estándares y criterios; y un ente interno (el programa o la institución) con un papel más pasivo, que se apega a las reglas establecidas y realiza un autoestudio tratando de complacer las demandas del organismo acreditador (Dobles, 1996).

- La cuarta generación es denominada por Guba y Lincoln (1989) como "constructiva", lo cual responde a la posición epistemológica desde la cual se realiza. También se 
denomina interpretativa o sensible, en el sentido de poder diseñar diferentes formas de enfocar una evaluación. Los límites y parámetros son construidos de manera conjunta entre las personas involucradas y los evaluadores, razón por la cual también se le denomina "evaluación emergente".

Según Dobles (1996), la evolución de la autoevaluación y acreditación como procesos evaluativos, implican a su vez una generación de cambio en la visión social educativa y en la visión de cómo se construye el conocimiento humano y del para qué se desea la autoevalución. Según la autora, se da el surgimiento de un paradigma alternativo donde el acreditado pasa de estar sometido a lo que el evaluador desea, con poca reflexión, a tener un papel más activo por cuanto se convierte en constructor y reconstructor de su propia realidad.

En este sentido, agrega Dobles (1996), la acreditación deberá otorgar tanta importancia al organismo externo como al interno, para lograr un acercamiento, una interacción, con el propósito de que entre todas las personas se construya un clima de reflexión del quehacer mismo, para encontrar soluciones creativas a los problemas.

La autora señala además, que esta situación significa un trastorno en el papel de la acreditación que pasaría de ser un ente fiscalizador y controlador, a un ente involucrado plenamente con los participantes en el proceso, donde se adquieren compromisos morales.

Ahora bien, teniendo presentes estos diversos puntos de vista, se observa con más claridad la relación entre propósito y enfoque. La autoevaluación para el mejoramiento, sin otra pretensión, suele ser un proceso de convencimiento interno por parte de quienes desarrollan programas o planes de estudio. Se caracteriza además por presentar diseños y períodos de tiempo irrestrictos, con un riesgo potencial de no concretar acciones o no finalizar los procesos sino existe un verdadero control metodológico.

Pero por otra parte, si la intención es la acreditación, con el elemento autorregulador de por medio, puede acontecer que la razón de la autoevaluación sea el producto del convencimiento de las personas responsables de una carrera o programa, pero también puede ser el producto del convencimiento de unos pocos, e incluso puede ser el producto de una decisión impuesta por una determinada persona. Desde esta perspectiva, el carácter acreditable de la autoevaluación la ubica esencialmente dentro de un enfoque tecnológico.

Ahora bien, el tránsito de la autoevaluación desde una tercera (enfoque tecnológico) hasta una cuarta generación (enfoque constructivo), puede hacer surgir una serie de procedimientos metodológicos diversos, híbridos entre enfoques y paradigmas, pero si realmente se desea llegar a una cuarta generación de la autoevaluación, se necesitará por 
parte de quienes se responsabilizan de ella, sean investigadores, evaluadores, comisiones o grupos, de una toma de conciencia clara y transparente respecto al papel que están asumiendo, la razón de ser de la autoevaluación y al cómo se llega al conocimiento de la realidad.

Será la toma de conciencia la que permitirá a la autoevaluación, tomar lugar bajo un enfoque "constructivo" y bajo un punto de vista "contextual o axiológico", para considerarla dentro del ámbito de la investigación.

\section{La autoevaluación transformada en investigación.}

Este primer ámbito tiene su origen en la opinión contraria de quienes afirman que los procesos de autoevaluación no constituyen procesos de investigación.

En el caso de la Escuela de Formación Docente, el equipo investigador, responsable del proceso de autoevaluación, enfrentó esta concepción como su primera metarreflexión. Este equipo fortaleció la creencia de que un proceso de autoevaluación puede ser llevado al plano de la investigación evaluativa. Conviene agregar que en los procesos regulares de autoevaluación, no es obligante señalar la posición paradigmática investigativa, ni tampoco explicitar el acercamiento epistemológico al que se recurre para lograr sus propósitos, quedando a criterio de las personas responsables de la autoevaluación, el señalar su marco investigativo de referencia.

Dentro de un contexto investigativo, la ausencia de un marco paradigmático de referencia, amplio y claro, ciertamente anula la posibilidad de que un proceso de autoevaluación sea llevado al plano de la investigación. De lo contrario entonces, la autoevaluación puede ser mejor comprendida como un proceso meramente técnico.

De tal manera surgen algunos cuestionamientos, necesarios de establecer, para poder determinar la transformación de la autoevaluación, como un proceso meramente técnico a un proceso investigativo. Ahora bien, sin pretender ofrecer respuestas exhaustivas, se presentan a continuación algunos de esos cuestionamientos con posibles respuestas, desde la visión, formación y experiencia de quien suscribe, como persona dedicada a la autoevaluación, investigación y diseño curricular.

- ¿Cuál es la diferencia entre una evaluación y una investigación evaluativa? Ambos procesos requieren resultados concretos, sin embargo, en una evaluación se concretan y aplican procedimientos, sean preescritos o no, para el logro de resultados que permitan la toma decisiones respecto a niveles de mejoramiento de una situación 
dada. Según la documentación consultada y la experiencia vivida, se puede afirmar que ésta es la posición asumida en la Universidad de Costa Rica, desde su ente responsable evaluativo, el Centro de Evaluación Académica. Esta posición es comprensible por cuanto dicho centro además de ser una instancia que investiga, es un medio de gestión y de control.

Por su parte la investigación evaluativa, cuenta con un marco de referencia, que la lleva al plano de la conciencia de su razón de ser; se ubica dentro de una posición paradigmática para entender y sustentar varios planos: los resultados, el procedimiento, las consecuencias y las proposiciones.

La evaluación conlleva en sí "valoraciones fundamentadas". La tarea básica de la investigación es "la producción de nuevos conocimientos".

En el caso de que la evaluación conduzca al interjuego entre comprensión y valoración, entonces ocurre una actividad de investigación, en la medida en que al realizar un análisis conceptual del objeto por evaluar y al construir una síntesis, tanto conceptual como teórica sobre él mismo, se está produciendo un conocimiento nuevo sobre tal objeto evaluado, tal y como lo señala de Alba (1991). Ahora bien ese conocimiento puede ser de muy diversa índole.

En síntesis, se puede concebir a la evaluación como una investigación evaluativa, en la medida de que en toda tarea de evaluación se conjugan aspectos teóricos (vinculados con la producción de conocimiento) y aspectos axiológicos (vinculados con la valoración y el compromiso).

- ¿Si la autoevaluación es evaluación, entonces es investigación?

- ¿Si la evaluación no es investigación, entonces la autoevaluación tampoco lo es?

- ¿Cuáles son las condiciones que hacen de la evaluación y por tanto de la "autoevaluación", procesos de investigación?

De hecho la autoevaluación es un estilo de evaluación, la diferencia estriba en que para la evaluación externa el compromiso del evaluador ante la situación y las personas es mucho menor. En un proceso de autoevaluación, los responsables tienen un alto grado de compromiso ante la situación, las personas se ven altamente involucradas y afectadas por el proceso y los resultados, es decir se ven implicadas. 
La autoevaluación como investigación evaluativa es propositiva, es decir, necesariamente conduce a obtener propuestas pertinentes para lograr una mejor condición de una realidad específica.

Dado lo anterior, la autoevaluación puede ser exclusivamente un proceso evaluativo, pero lejos de limitarse a ello, puede trascender al plano de la investigación. Es aquí cuando el investigador toma un papel primordial.

Como ejemplo de lo anterior, se señala el caso del proceso de autoevaluación en la Escuela de Formación Docente, el cual, desde una visión institucional se considera como un proceso evaluativo más, sin embargo desde la visión de su equipo investigador, el proceso fue llevado al plano de la investigación, por cuanto, no sólo se buscó el alcance de resultados y propuestas de autorregulación con miras a la acreditación, sino al esclarecimiento de su posición paradigmática, para trascender a hacia una cultura de mirarse a sí mismos y alcanzar una mejor conformación de lo propuesto como imagen en su Proyecto Pedagógico. Es así que en el caso de la Escuela de Formación Docente la autoevaluación fue llevada al plano de la investigación evaluativa por cuanto:

- Se presentó un acercamiento desde el punto de vista paradigmático de la investigación.

- Se explicitó detalladamente, no sólo los resultados sino el proceso metodológicamente alcanzado.

- Se develaron las reflexiones, no sólo de los sujetos involucrados, sino del equipo responsable, que de hecho fueron parte de los sujetos involucrados.

- Se ofreció, y así está estipulado dentro de los objetivos, insumos para la constitución de un modelo de autoevaluación, más allá de la simple indicación de factores, criterios e indicadores por tomar en cuenta.

Este insumo se concreta por medio de la caracterización del modelo de autoevaluación, utilizado en la Escuela de Formación Docente, punto casi obligado a retomar como consecuencia de la investigación. Este aspecto representa un insumo tanto en el nivel investigativo como para el Centro de Evaluación Académica, instancia que regula los procesos de autoevaluación en la Universidad de Costa Rica, así como para la propia Escuela de Formación Docente. Esta caracterización será objeto de atención en un posterior artículo. 


\section{La autoevaluación como segundo objeto de investigación.}

El objetivo número tres de la investigación para la autoevaluación realizada en la Escuela de Formación Docente: retroalimentar con insumos para la constitución de un modelo de autoevaluación; configuró al "proceso de autoevaluación" como un segundo objeto de estudio. Por ello el desarrollo del proceso de autoevaluación y su reflexión se constituyeron en estrategias fundamentales.

\section{Una posición paradigmática de investigación para la autoevaluación.}

La ausencia de un paradigma explícito que respalde la ejecución de la autoevaluación puede hacer pensar en ésta como un proceso acientífico y neutro, sin embargo no es así, ya que posteriormente se entrará en un análisis del proceso evaluativo desde el punto vista de premisas naturalistas y positivistas, que harán ver la posición del proceso autoevaluativo.

El carácter acientífico y neutro se disipan, por tres razones principalmente: en primer término, al comprender que la autoevaluación es en sí una evaluación; segundo, que la autoevaluación para la acreditación en Costa Rica, corresponde a una evaluación de tercera generación, por cuanto centra su atención en criterios estándares. Y tercero, la autoevaluación recae en un enfoque "tecnológico" puesto que se pretende validar y legitimar.

La literatura consultada no aborda la autoevaluación directamente como "investigación", se aborda desde la perspectiva de la evaluación. En este sentido la misma literatura, manifiesta, una preocupación por el aspecto conceptual de la evaluación, el para qué, el cómo obtener resultados, más no una preocupación del cómo se aborda el conocimiento desde la evaluación y cómo se sistematiza el proceso, de manera tal que permita su propia reflexión, ya que por lo general existe una tendencia a la prescripción metodológica, o al menos, una prescripción de elementos por considerar, esto en el caso dado, especialmente, de que la intención posterior sea la acreditación.

Concretamente, la autoevaluación en la Escuela de Formación Docente, evidenció una posición paradigmática que transitó entre lo naturalista y positivista, contrario a lo que podrían pensar algunos teóricos de la investigación. A continuación se justifica esta posición:

- Según la intencionalidad: existen dos niveles una inmediata y otra mediata. La primera intención buscó autoevaluarse para mejorar y ser más coherentes con el Proyecto Pedagógico, lo cual ubica a la autoevaluación dentro de una posición más naturalista. La segunda intención paralela, es la acreditación, señalada como acuerdo de Asamblea de 
Escuela y asumida como meta del proceso de autoevaluación. Esto ubica a la autoevaluación dentro de una perspectiva positivista, al responder a un enfoque tecnológico, para legitimar.

- Por el papel y lugar que ocupa el equipo investigador: se presenta una ubicación dentro de un paradigma naturalista, por cuanto las investigadoras constituyeron parte de los sujetos que conforman la historia misma, y fueron parte del objeto mismo en estudio. Al ser parte de los sujetos y el objeto mismo, sus creencias y valores determinan un proceso metodológico y una valoración irrepetibles en cualquier otro contexto.

Por el planteamiento metodológico: concebida como investigación, la autoevaluación de planes de estudio, implicó un complejo planteamiento metodológico, por cuanto el análisis de la realidad por retratar y comprender, no se redujo al establecimiento de una lógica analítica cuantitativa de lo que se produce (hay/no hay-más/menos), ya que para observar al objeto en estudio se requirió además, de una perspectiva desde el punto de vista de lo cualitativo.

Desde lo metodológico, la autoevaluación se mira complejamente en fluctuación entre lo cualitativo y cuantitativo, al considerar a las unidades académicas, gestoras de planes de estudio en desarrollo, como universos medibles para llegar a entenderlos como universos de significados o viceversa, y así, del plano del significado llegar al plano medible. No se trató, en el caso que nos ocupa, de un tratamiento metodológico simple de incluir técnicas cualitativas o cuantitativas, sino de una integración de las mismas. Se trató de la capacidad de las investigadoras para establecer articuladamente una relación que evidenciara, no sólo una imagen socializada del objeto de estudio, sino una valoración de esa imagen o configuración, entendiendo por valoración, según de Alba (1991) el logro de una perspectiva particular respecto al objetivo de estudio.

Por la dinámica de los planes de estudio: los planes de estudio son objetos interactuantes en la unidad académica, cuya dinámica interna está establecida por los sujetos que la integran (estudiantes, docentes, administrativos, directores) y la cultura propia que se establece, de acuerdo con los supuestos filosóficos, epistemológicos, axiológicos, metodológicos; de carácter grupal e individual. Pero además esa dinámica está determinada, a su vez, por otras dinámicas externas y por condicionantes institucionales de carácter operativo, administrativo, político y conceptual.

El objeto de estudio comprende el desarrollo de planes de estudio dentro de la Escuela de Formación Docente, por tanto, lograr una perspectiva respecto a ello significa 
alcanzar un modo, o modos intencionales de tener conciencia de ese "desarrollo", con un relativo buen nivel de certidumbre, con todo y los límites metodológicos producidos como la poca profundización en el establecimiento de algunas relaciones y el manejo de los niveles de participación que, en gran medida queda en el plano de la participación colaborativa.

Ahora bien, a continuación se ubica la experiencia desarrollada de autoevaluación como investigación evaluativa dentro de la dimensión paradigmática investigativa. Para lograr este propósito se presentan las posiciones paradigmáticas de la investigación, naturalista y positivista, según sus premisas y determinados autores. 
Tabla 1 Acercamiento paradigmático investigativo de la autoevaluación con miras a la acreditación en la Escuela de Formación Docente

PREMISAS NATURALISTAS, según Dobles, Zúñiga y García, 1996)

Premisas ontológicas: Están relacionadas con la perspectiva de la realidad (Pérez, 1990). Existen múltiples realidades, construidas holística e interrelacionadamente (Dobles y otros, 1996)

\section{CÓMO SE EVIDENCIA:}

Desde esta perspectiva existe una multiplicidad de realidades no estáticas que se influyen unas a otras:

- Una realidad institucional, con variados ámbitos que inciden en la realidad general del desarrollo pedagógico de la Escuela de Formación Docente

- Una realidad general: la Escuela de Formación Docente, influenciada desde el exterior (realidad institucional) e influenciada desde abajo hacia arriba por las realidades de cada carrera.

- La realidad de una comunidad de docentes que comparten una visión de unidad académica, que comparten algunos dos o tres carreras y por tanto influencian su quehacer hacia un lado y otro. Pero también tienen visiones individuales de lo que es o se debe ser. Esta comunidad docente se ve influencia por la comunidad estudiantil.

- Una comunidad estudiantil, que aunque diferente para cada carrera, tiene espacios comunes y se ve influenciada por el desarrollo pedagógico de una carrera e influenciada por la comunidad docente. Cada población de cada carrera manifiesta visiones de lo que es o debe ser cada carrera.

- Una realidad académica: un tronco común que inevitablemente incide en las tres realidades de tres carreras.

- Una cultura administrativa, gestada por la influencia de todos los actores: administrativos, directores, estudiantes, docentes, la cual influye en diversos ámbitos de quehacer de la escuela.

- Una realidad del equipo investigador, que al ser parte de la cultura y el desarrollo pedagógico de la escuela, muestra una visión desde el entorno mismo y no desde el exterior, compartiendo criterios o difiriendo de ellos.
PREMISAS POSITIVISTAS, según Contreras (1998)

Premisas ontológicas: Una única realidad, tangible, que puede fragmentarse en variables y procesos independientes. La investigación se puede controlar y predecir.

\section{CÓMO SE EVIDENCIA:}

Desde esta perspectiva puede verse una única realidad: el desarrollo pedagógico de la Escuela de Formación Docente. Esta realidad puede verse fragmentarse en tres realidades:

- El desarrollo de la carrera del Bachillerato en Educación Preescolar.

- El desarrollo de la carrera del Bachillerato en Educación Primaria.

El desarrollo del Núcleo Pedagógico para las Enseñanzas en Secundaria.

A su vez, estas realidades pueden fragmentarse en factores: plan de estudio, docencia, población estudiantil, organización-administración, recursos físicos y financieros.

Hay un cierto nivel de control, mediante el uso de criterios e indicadores que determinan lo que hay que autoevaluar, sin embargo, los resultados no se pueden controlar. En alguna medida la aplicación y el procesamiento de los datos estadísticos es controlable.

Desde esta perspectiva se han generado tres informes divergentes.
Premisas epistemológicas: Implican el cómo se accede al conocimiento. En este sentido, sujeto y objeto de estudio interactúan y son inseparables (Dobles y otros, 1996). El sujeto y objeto de la investigación tienen una influencia recíproca a través de estrategias didácticas.
Premisas epistemológicas. El sujeto y el objeto son independientes. Hay una dualidad entre investigador y conocimiento. Al conocimiento del objeto se llega desde el exterior. 
PREMISAS NATURALISTAS, según Dobles, Zúñiga y García, 1996)

COMO SE EVIDENCIA

El conocimiento, en esencia, fue dado desde lo interno, por varias razones:

- El equipo investigador es parte de la cultura y el desarrollo de la Escuela de Formación Docente. Cada investigadora contaba con 20 años o más de laborar en la unidad académica, conociendo el funcionamiento de la unidad acádemica y del desarrollo de los planes de estudio en mayor o menor medida.

- El acceso a la información fue logrado de lo particular a lo general: conocimiento interno de documentación propia de planes de esttudio y unidad académica, para posteriormente ir logrando documentación y conocimiento del contexto institucional. Las útimas consultas fueron a instancias externas a la unidad académica.

- La validación e interpretación de información por parte de docentes fueron logrados por intermedio del equipo investigador; para conseguir un cuerpo de valoración conjunto, sobretodo el establecimiento de fortalezas y debilidades. Fue un proceso muy mediatizado, no tanto así el logro de la información.
PREMISAS POSITIVISTAS, según Contreras (1998)

\section{COMO SE EVIDENCIA}

Aunque el procedimiento estableció un conocimiento desde lo interno, sí se dio intencionalidad de establecer distancia entre investigador y conocimiento mediante la estrategia de responsabilizar a cada investigador de la profundización en el análisis de aquel plan de estudio que menos conocía, esta estrategia tuvo la intención de evitar las interpretaciones segadas, sin fundamentación.

Esta estrategia funcionó, pero el conocimiento previo e interno siempre estuvo presente.

Se dieron momentos de distancia entre sujetos participantes (docentes y estudiantes) y conocimiento, de la realidad.

\begin{abstract}
Premisas heurísticas: Se refieren a la manera o camino a través del cual se alcanza el objetivo. No se generaliza, solo se desarrollan postulados. Las diferencias y peculiaridades las determina el contexto. No interesa las relaciones de causa y efecto (Dobles y otros, 1996).
\end{abstract}

CÓMO SE EVIDENCIA: el conocimiento es irrepetible, los resultados son únicos para un espacio y tiempo determinados, la situación de los planes de estudio es irrepetible para otros planes de estudio y otros momentos. Cada plan de estudio es particular, contiene su propia contextualización, pero a la vez se comparten. Las características de cada plan de estudio se dan por efectos multifactoriales, de elementos que coexisten. Hay resultados multifactoriales, no generalizables.

Premisas axiológicas: La investigación se determina por los valores del investigador, del paradigma, el contexto y de la teoría en que se fundamenta. CÓMO SE EVIDENCIA:

Una condición primordial del proceso de autoevaluación, es ofrecer valoraciones fundamentadas. Es necesario utilizar la información e interpretación para ofrecer valoraciones que determinen las fortalezas y debilidades de la unidad académica y de los planes de estudio.

Se ofrecen valoraciones, basadas en la información descriptiva, es decir, el alcance de una perspectiva, con un alto nivel de certidumbre, respecto a los
Premisas heurísticas: Cuerpo nemotécnico de conocimientos Se producen leyes y generalizaciones que constituyen afirmaciones verdaderas. Aplicables a cualquier lugar o tiempo.

Se establecen relaciones de causa-efecto. El efecto se produce por una causa que precede temporalmente o simultáneamente al efecto.

CÓMO SE EVIDENCIA: Se establecen resultados de causa y efecto, para lo cual se utilizan básicamente datos estadísticos. Por ejemplo, a menor producción académica, menor impacto al exterior, a mayor producción académica, mayor impacto al exterior.

También existe información generalizable, de carácter normativo repetible para todos los planes de estudio, pero no necesariamente aplicable de igual manera.

Continúa... Premisas axiológicas: Los conocimientos están exentos de valoraciones, de ahí el uso de una metodología objetiva. COMO SE EVIDENCIA:

Existe un cuerpo amplio de información descriptiva.

No todas las valoraciones son dadas por triangulación.

Existen factores, criterios e indicadores predeterminados, que evitan en alguna medida hacer interpretaciones fuera de los efectos posteriores, el logro de una de las metas establecidas: "la acreditación" 
PREMISAS NATURALISTAS, según Dobles, Zúñiga y García, 1996)

planes de estudio en el contexto de la unidad académica. Hay interpretaciones no predecibles que se dan como producto de espacios de triangulación.

Los valores de los investigadores determinan interpretaciones por cuanto pertenecen y participan del desarrollo de los planes de estudio mismos y de la dinámica estructural en la cual se ubican.

Los valores del paradigma han sido difíciles de establecer, por cuanto inicialmente no existe una adopción explícita por un paradigma investigativo.

Los valores teóricos iniciales son dados por la concepción de "investigación evaluativa" y el concepto de "autoevaluación"

Aún desde una perspectiva más lejana "la acreditación", y aunque los pares externos conocen desde el exterior, siempre se ofrece una valoración.
PREMISAS POSITIVISTAS, según Contreras (1998)

La información lograda debe ser tal que permita a "pares externos", alcanzar un conocimiento de los planes de estudio desde el exterior, sin establecer valoraciones previas.

La metodología establece el uso de técnicas no sólo cualitativas, como el uso de la entrevista, las plenarias, los talleres, sino el empleo de cuestionarios para el logro de información estadística, especialmente en evaluación de cursos y conocimiento de planes de estudio por parte de los estudiantes. 


\section{Consideraciones finales}

1. Abordar el proceso de autoevaluación de manera investigativa, demandó un estudio y análisis de dos objetos de estudio: la intención de la autoevaluación y el cómo se hace. Este doble objeto de análisis, permite identificar la posición paradigmática para finalmente tomar conciencia del papel del investigador y de las personas involucradas, asunto que aún está en reflexión.

2. Queda de manifiesto que la autoevaluación, puede ser abordada desde una perspectiva investigativa, en cuyo caso se hace necesario un marco de referencia que permita ser contrastado frente a la sistematización de lo metodológico.

3. Queda también de manifiesto, que la autoevaluación puede ocurrir en un entorno de fluctuación entre lo naturalista y positivista. Sin embargo, del acercamiento paradigmático realizado, quedan pendientes dos aspectos: un mayor esclarecimiento del aporte que ofrece la fluctuación paradigmática al mejoramiento interno del desempeño de las carreras y qué tan pertinente resulta la fluctuación paradigmática respecto al proceso de acreditación.

4. Dado el anterior punto, las actitudes críticas y autocríticas debieran ser fomentada por aquellas instancias universitarias sobre las cuales recae el papel de asesoría en procesos semejantes.

5. Quedan las verdaderas intenciones de la autoevaluación con miras a la acreditación, en el contexto de una universidad pública ¿cuáles son las intenciones y cuáles deberían ser?:

- Legitimar su quehacer académico.

- Garantizar un alto nivel de calidad a lo interno de la institución.

- Garantizar un alto nivel de calidad frente a universidades privadas.

- Garantizar una respuesta pertinente frente a las necesidades y demandas sociales de tal manera que se logre una transformación con miras al bien común, definidas éstas de manera estatutaria.

6. Del proceso de autoevaluación vivenciado, y del análisis paradigmático realizado, desde la perspectiva de quien suscribe, existe una clara visión de que las intenciones de la autoevaluación son legitimar y garantizar un cierto nivel de calidad a lo interno de la institución, pero no así de las otras dos intenciones, en menor medida aquella que llama a garantizar una respuesta a las demandas y necesidades sociales, con miras a una transformación de la sociedad. Esto por cuanto a pesar de contar con criterios establecidos de valoración, se carece de un perfil o cuerpo de competencias 
profesionales sobre el cual establecer una valoración de contraparte, pero se carece además de un verdadero seguimiento a graduados que permita develar el verdadero desempeño e impacto que generan en sus espacios laborales.

7. Quedan por tanto en consideración, otros cuestionamientos: acreditar qué, respecto a qué y para qué.

8. La mayor pretensión en un futuro será trascender de una autoevaluación de tercera generación a una de cuarta generación, sin embargo será necesario madurar los procesos de tal manera que sea el momento histórico el que ofrezca las condiciones para que dicha evolución se produzca.

\section{REFERENCIAS}

Cook, T.D. y Reichardt, Ch, S. (1997). Métodos cualitativos y cuantitativos en investigación evaluativa. Madrid: Morata.

Contreras, I. (s.f.). La investigación Etnográfica en el Marco de la Investigación Cualitativa en Educación. Material poligrafiado.

De Alba, A. (1991). Evaluación Curricular. Conformación conceptual del campo. México: Centro de Estudios sobre la Universidad de México.

Díaz S., M. (1996). Aplicación de un diseño de Investigación Evaluativa para el plan de Estudios de Currículo de Licenciatura en Administración de Empresas Turísticas. Tesis de Maestría. Universidad Católica de Santa María de la Antigua. Panamá.

Díaz Barriga, A. (1996). Evaluación Académica: Organismos Internacionales y Política educativa. En Memoria Foro Taller Internacional sobre Tendencias Actuales en la Medición y Evaluación Educativa. Compiladora Leda Badilla. Costa Rica: Universidad de Costa Rica.

Dobles, M. C.; Zúñiga, M., García, J. (1996). Investigación en educación: procesos interacciones construcciones. Costa Rica: EUNED

Dobles, C. (1996). Acreditación en Educación Superior: Orígenes, prácticas actuales, perspectivas. En Memoria Foro Taller Internacional sobre Tendencias Actuales en la Medición y Evaluación Educativa. Compiladora Leda Badilla. Universidad de Costa Rica.

Guba, E. y Lincoln, Y. (1989). Fourth Generation Evaluation. California: SAGE Pub. Incorporated.

Sistema Nacional de Acreditación de la Educación Superior, SINAES. (2000). Manual de Acreditación. San José, Costa Rica: SINAES.

Universidad de Costa Rica. (1999). Guía de Autoevaluación. San José, Costa Rica: Centro de Evaluación Académica. Universidad de Costa Rica. 